\title{
INVESTIGATING A NOVEL MODIFIABLE FACTOR AFFECTING RENAL FUNCTION AFTER PARTIAL NEPHRECTOMY: CORTICAL RENORRHAPHY
}

\author{
Anna Fenner and Clint Bahler, MD \\ Indiana University School of Medicine
}

\begin{abstract}
Background:
Partial nephrectomy is a common treatment for the removal of renal masses. Typically, during the procedure, stitches are used to close two layers of the kidney-both deep and superficial. Renorrhaphy of the superficial layer, thought to reduce the risk of bleeding and urine leak, is routinely performed but has not been sufficiently studied.
\end{abstract}

Hypothesis:

Cortical renorrhaphy is a modifiable factor affecting renal function after partial nephrectomy. Omitting this step will preserve renal parenchyma without significantly increasing complications.

\section{Methods:}

A randomized, controlled trial is underway. Interim statistical analysis has been performed on the data being collected. Patients underwent partial nephrectomy with or without cortical renorrhaphy according to their randomized group assignment. Three-dimensional models were constructed using semi-automatic segmentation planimetry of the kidney prior to surgery and at 4-months after tumor resection to determine volume loss in the operated kidney.

\section{Results:}

The median (range) volume loss in the non-renorrhaphy group $(n=8), 13 \%(0-24 \%)$, was trending lower than the renorrhaphy group $(n=8), 22 \%(12-39)$. Using multiple linear regression, experimental group $(p=0.0808)$ and warm-ischemia time $(p=0.0995)$ were significant at the 0.1 level. Tumor size was not statistically significant $(p=0.2644)$. There was one Clavien 3 complication in each group: The renorrhaphy group had one urine leak requiring a drain, and the non-renorrhaphy group had one postoperative bleed requiring selective embolization. Demographics were comparable among the two groups with both having 4 white males and 4 white females. The mean age (58 and 55 for renorrhaphy and non-renorrhaphy, respectively) and tumor size were also comparable.

\section{Conclusion and impact:}

A trend of increased volume loss from cortical renorrhaphy is seen as predicted by retrospective data. Completion of the trial is needed to conclude whether this is statistically significant. 